\title{
PEDERSEN IDEAL AND GROUP ALGEBRAS
}

\author{
KLAUS HARTMANN
}

\begin{abstract}
ABSTRACr. For a locally compact $T_{2}$ group $G$ which has an open subgroup of polynomial growth (e.g., $G$ a group that has a compact neighbourhood invariant under inner automorphisms or $G$ a compact extension of a locally compact nilpotent group) the intersection of the Pedersen ideal of the group $C^{*}$-algebra with $L^{1}(G)$ is dense in $L^{1}(G)$ (Theorem 1). For groups with small invariant neighbourhoods this intersection is the smallest dense ideal of $L^{1}(G)$, and it consists exactly of those $f \in L^{1}(G)$ whose "Fourier transform" vanishes outside some (closed) quasicompact subset of $\hat{G}$ (Theorem 3); the Pedersen ideal of $C^{*}(G)$ is described as the set of all $a \in C^{*}(G)$ for which $\{\pi \in \hat{G}: \pi(a) \neq 0\}$ is contained in some (closed) quasicompact subset of $\hat{G}$ (Theorem 2 ).
\end{abstract}

1. Introduction. In [11] G. K. Pedersen proved that every $C^{*}$-algebra $A$ has a smallest dense order-related ideal $K_{A}$, and in $1975 \mathrm{~K}$. B. Laursen and A. M. Sinclair showed that $K_{A}$ (the so-called Pedersen ideal of $A$ ) is the smallest ideal among all dense ideals of $A$ [5]. In [12] Pedersen asked whether $K_{G} \cap L^{1}(G)$ is dense in $L^{1}(G)$ where $G$ is a locally compact $T_{2}$ group, $L^{1}(G)$ its group algebra and $K_{G}$ the Pedersen ideal of the group $C^{*}$-algebra $C^{*}(G)$. The answer is affirmative in the case where $G$ is abelian or compact (well known) or a connected real nilpotent Lie group [13].

In this note we shall show that the answer is affirmative even in the case where $G$ has at least one compact neighbourhood $U$ of the group identity such that $\lambda\left(U^{k}\right)=O\left(k^{n}\right)$ for some fixed $n \in N$ ( $\lambda$ left Haar-measure). Examples of such groups are, e.g., groups that contain an open subgroup which is a compact extension of a (locally compact) nilpotent group, and IN-groups ( $G \in[\mathrm{IN}] \leftrightarrow G$ has a compact invariant neighbourhood); the latter because the open subgroup $G_{F}$ consisting of all elements with relatively compact conjugacy classes has polynomial growth [10].

In the special case of a SIN-group $G(G \in[\mathrm{SIN}] \leftrightarrow G$ has a fundamental system of compact invariant (under inner automorphisms) neighbourhoods of the identity of $G$ ) we show that $K_{G}$ consists exactly of those $a \in C^{*}(G)$ for which $\{\pi \in$ $\hat{G} ; \pi(a) \neq 0\}$ is contained in a quasicompact subset of $\hat{G}$, and that $L^{1}(G) \cap K_{G}$ is the smallest dense ideal of $L^{1}(G)$.

The question whether there is a locally compact group $G$ at all for which $L^{1}(G) \cap K_{G}$ is not dense in $L^{1}(G)$ or even $L^{1}(G) \cap K_{G}=\{0\}$ still seems to be open.

Received by the editors March 14, 1980 and, in revised form, December 5, 1980.

1980 Mathematics Subject Classification. Primary 22D15; Secondary 22D25, 43A20.

Key words and phrases. Group algebra, group $C^{*}$-algebra, Pedersen ideal, polynomial growth, functional calculus, SIN-group. 
ReMARK. The existence of a smallest dense ideal in $L^{1}(G), G \in[\operatorname{SIN}]$, has also been shown in [4].

2. Pedersen ideal and groups of polynomial growth. Let $A$ be a $C^{*}$-algebra. The Pedersen ideal $K_{A}$ can be obtained in the following way (see [12]): $K_{A}$ is the complex linear span of the invariant face generated by the set

$$
K_{A}^{+\infty}:=\left\{x \in A^{+}: \exists y \in A^{+} \text {with } x=x y\right\} .
$$

(A face $F$ is a convex cone in $A^{+}$such that: $x \in F, z \in A^{+}, z<x \Rightarrow z \in F$; $F$ is called invariant $\Leftrightarrow a^{*} F a \subseteq F \quad \forall a \in A \Leftrightarrow u^{*} F u=F \quad \forall u \in \tilde{A}, u$ unitary, $\tilde{A}$ the $C^{*}$-algebra obtained by adjunction of a unit (if $A$ does not have a unit)).

For group algebras of groups with polynomial growth, J. Dixmier has found in [1] a functional calculus which turns out to be a very useful tool in harmonic analysis (see e.g. [7] and [8]).

Let $C_{n}(n \in \mathrm{N})$ denote the set of all functions $\varphi: \mathbf{R} \rightarrow \mathbf{C}, \varphi(0)=0$ which have continuous and integrable derivatives of order $\leqslant n+3$. Let $V$ be a compact neighbourhood of the identity of a locally compact group $G$ such that $\lambda\left(V^{k}\right)=$ $O\left(k^{n}\right), f=f^{*} \in L^{1}(G) \cap L^{2}(G)$ such that $f=0$ outside $V$. Then for every $\varphi \in C_{n}$ the integral

$$
\varphi\{f\}:=\frac{1}{2 \pi} \int_{\mathbf{R}} \exp (i \lambda f) \hat{\varphi}(\lambda) d \lambda
$$

converges in $L^{1}(G)$ ( $\hat{\varphi}$ is the Fourier transform of $\varphi$; "exp" is with respect to convolution) and for every *-representation $\pi$ of $L^{1}(G)$ on a Hilbert space

$$
\pi(\varphi\{f\})=\varphi(\pi(f))
$$

where the right side is defined by the usual operational calculus on the hermitian operator $\pi(f)$.

THEOREM 1. Let $G$ be a locally compact $T_{2}$ group with a compact neighbourhood $V$ of the identity $e$ of $G$ such that $\lambda\left(V^{k}\right)=O\left(k^{n}\right)$ for some $n \in N$ (equivalently: $G$ contains an open subgroup of polynomial growth). Then $L^{1}(G) \cap K_{G}$ is a dense ideal in $L^{1}(G)$ (where $K_{G}$ denotes the Pedersen ideal of $C^{*}(G)$ ).

Proof. Take a compact neighbourhood $U=U^{-1}$ of $e$ such that $U^{2(n+4)} \subseteq V$, $\left(g_{i}\right)_{i \in I}$ a bounded approximate unit for $L^{1}(G), g_{i}: G \rightarrow \mathbf{R}$ continuous, $g_{i}(x)>0$ $\forall x \in G, \operatorname{supp}\left(g_{i}\right) \subseteq U,\left\|g_{i}\right\|_{1}=1$, and define $f_{i}:=g_{i}^{*} * g_{i}$. Take $\varphi \in C_{n}$ such that

$$
\begin{aligned}
& \varphi(t)=t^{n+4} \quad \text { for all } t \text { with }|t|<1=\left\|f_{i}\right\|_{1}, \\
& \varphi(t) \geqslant 0 \quad \forall t \geqslant 0 .
\end{aligned}
$$

Now fix $i$, choose $\varepsilon>0$. There is a real valued $\psi_{i, e} \in C_{n}$ with

$$
\begin{aligned}
\psi_{i, \mathrm{e}}(t)=\varphi(t) & \forall t \in \mathbf{R} \backslash[-1,+1], \\
\left|\psi_{i, \varepsilon}^{(\alpha)}(t)-\varphi^{(\alpha)}(t)\right|<\varepsilon / A_{i} & \forall t \in[-1,+1], \alpha=0,1, \ldots, n+3,
\end{aligned}
$$

and

$$
\psi_{i, e}(t)=0 \quad \forall|t|<\delta_{i, e} \quad \text { for some } \delta_{i, e} \in(0,1)
$$


[1, Lemme 8] where $A_{i}$ is the constant $A$ (independent of $\varepsilon$ ) in the proof of [1, Théorème 1.b]. Without loss of generality $\psi_{i, \mathrm{e}}(t)>0 \forall t>0$.

Now we have $\varphi\left\{f_{i}\right\}=f_{i}^{n+4}$ (exponent with respect to convolution) and

$$
\left\|\psi_{i, \varepsilon}\left\{f_{i}\right\}-f_{i}^{n+4}\right\|_{1}<\varepsilon
$$

[1, Théorème 1.b], hence $\left\{\psi_{i, \varepsilon}\left\{f_{i}\right\}: i \in I, 0<\varepsilon<1\right\}$ is a bounded approximating set for $L^{1}(G)$. If we can show all $\psi_{i, e}\left\{f_{i}\right\}$ to be in $K_{G}$ the proof is finished, since $\left\{f * \psi_{i, e}\left(f_{i}\right\}: f \in L^{1}(G), i \in I, 0<\varepsilon<1\right\}$ is dense in $L^{1}(G)$.

By construction $f_{i} \in C^{*}(G)^{+}$, and its spectrum $\sigma_{C^{*}(G)}\left(f_{i}\right)$ is contained in $[0,1]$. Since $\psi_{i, e}\left(f_{\mathrm{i}}\right)=\psi_{i, \mathrm{e}}\left\{f_{i}\right\}$ (take for $\pi$ the universal representation in $(*) ; \psi_{i, e}\left(f_{i}\right)$ means the usual functional calculus for $C^{*}$-algebras) and $\psi_{i, e}(t)>0 \forall t>0$, we have $\psi_{i, e}\left(f_{i}\right) \in C^{*}(G)^{+}$. Choose a function $\rho_{i, e}: \mathbf{R} \rightarrow[0, \infty), \rho_{i, e} \in C_{n}$ such that

$$
\rho_{i, e}(t)=1 \quad \forall t \in\left[\delta_{i, e}, 1\right]
$$

and

$$
\rho_{i, e}(t)=0 \quad \forall t \in \mathbf{R} \backslash\left[1 / 2 \cdot \delta_{i, e}, 2\right] .
$$

Now $\rho_{i, e}\left\{f_{i}\right\} \in L^{1}(G), \quad \rho_{i, e}\left\{f_{i}\right\}=\rho_{i, e}\left(f_{i}\right) \in C^{*}(G)^{+}$and $\psi_{i, e}\left(f_{i}\right) \rho_{i, e}\left(f_{i}\right)=\psi_{i, e}\left(f_{i}\right)$, hence $\psi_{i, e}\left\{f_{i}\right\} \in K_{G}^{+00} \subseteq K_{G}$.

REMARK 1. The properties of the bounded approximating set in the proof of Theorem 1 show at once that $I \cap K_{G}$ is dense in $I$ for every left (or right) ideal in $L^{1}(G)$ ( $I$ not necessarily closed).

Remark 2. The construction of the Pedersen ideal $K_{A}$ of a $C^{*}$-algebra $A$ shows at once that for all elements $x \in K_{A}$ the set $\{\pi \in \hat{A}: \pi(x) \neq 0\}$ is contained in a quasicompact subset of $\hat{A}$ since $a b=b\left(a, b \in A^{+}\right)$implies $\{\pi \in \hat{A}: \pi(b) \neq 0\} \subseteq$ $\{\pi \in \hat{A}:\|\pi(a)\| \geqslant 1\}$. Hence we have the following

Corollary. $G$ as in Theorem 1. The set of all $f \in L^{1}(G)$ for which the "Fourier transform" $\hat{f}, \hat{f}(\pi):=\pi(f), \pi \in \hat{G}$, vanishes outside a quasicompact set of $\hat{G}$ is dense in $L^{1}(G)$.

3. Pedersen ideal and SIN-groups. For SIN-groups we get more detailed information than in the corollary above:

TheORem 2. Let $G \in[S I N], K_{G}$ the Pedersen ideal of $C^{*}(G), J_{G}:=\left\{a \in C^{*}(G)\right.$; $\hat{a}$ vanishes outside a quasicompact subset of $\hat{G}\}$ (where $\hat{a}(\pi):=\pi(a) \forall \pi \in \hat{G})$. Then $J_{G}=K_{G}$.

Proof. We only have to show $J_{G} \subseteq K_{G}$. Consider the following mappings $t$ and $p:$

$$
\begin{aligned}
& t: \hat{G} \rightarrow \operatorname{Prim} C^{*}(G), \quad \pi \mapsto \operatorname{ker} \pi, \\
& p: \operatorname{Prim} C^{*}(G) \rightarrow G-\operatorname{Max} C^{*}\left(G_{F}\right) \cong E\left(G_{F}, G\right), \quad P \mapsto P \cap C^{*}\left(G_{F}\right),
\end{aligned}
$$

where $G$-Max $C^{*}\left(G_{F}\right)$ denotes the ideals of $C^{*}\left(G_{F}\right)$ which are maximal among the $G$-invariant modular ideals; $G$-Max $C^{*}\left(G_{F}\right)$ with hull-kernel topology is homeomorphic to $E\left(G_{F}, G\right)$ (the extreme points of the set of all $G$-invariant continuous positive definite functions $\gamma$ on $G_{F}$ with $\gamma(e)=1$ ) with the topology of compact 
convergence; the homeomorphism

$$
\begin{aligned}
& E\left(G_{F}, G\right) \rightarrow G-\operatorname{Max} C^{*}\left(G_{F}\right) \text { is given by } \\
& \gamma \mapsto\left\{a \in C^{*}\left(G_{F}\right):\left\langle a^{*} a, \gamma\right\rangle=0\right\} \quad[9,(4)] .
\end{aligned}
$$

For each $P \in$ Prim $C^{*}(G)$ there is a continuous positive definite indecomposable function $\varphi, \varphi(e)=1$ with $P=\operatorname{ker} \pi_{\varphi}$, and $P \cap C^{*}\left(G_{F}\right)$ corresponds to $\left(\varphi \mid G_{F}\right)^{G} \in$ $E\left(G_{F}, G\right)$, which is defined by

$$
\left(\varphi \mid G_{F}\right)^{G}(n):=\int_{\overline{I\left(G_{F}, G\right)}} \varphi\left(\beta^{-1}(n)\right) d \beta
$$

where $\overline{I\left(G_{F}, G\right)}$ is a compact group: the closure of the restrictions to $G_{F}$ of the inner automorphisms of $G$. The mapping $p: \operatorname{ker} \pi_{\varphi} \mapsto\left(\varphi \mid G_{F}\right)^{G}$ is well-defined from Prim $C^{*}(G)$ onto $E\left(G_{F}, G\right)$ (even continuous and proper). See [2].

Now take an arbitrary $a \in J_{G}$, choose $L \subseteq$ Prim $C^{*}(G)$ quasicompact with $t^{-1}(L) \supseteq\{\pi \in \hat{G}: \pi(a) \neq 0\}$. Since the "Fourier transform" of the hermitian and positive parts of $a$ vanish outside $t^{-1}(L)$ too, $a \geqslant 0$ without loss of generality. Since the algebra of functions in $L^{1}\left(G_{F}\right)$ that are central in $L^{1}(G)$ is a completely regular Banach algebra with maximal ideal space $E\left(G_{F}, G\right)$ (see $[3,(4)]$ or $\left.[6,(2.4)]\right)$ we can get $f \in L^{1}\left(G_{F}\right)$, central in $L^{1}(G)$, with

$$
\begin{aligned}
\hat{f}(\alpha) & :=\int_{G_{F}} f(x) \alpha(x) d x>0 \quad \forall \alpha \in E\left(G_{F}, G\right), \\
\hat{f}(\alpha) & =1 \quad \forall \alpha \in p(L) \subseteq E\left(G_{F}, G\right) .
\end{aligned}
$$

Then $f \in C^{*}\left(G_{F}\right)^{+} \subseteq C^{*}(G)^{+}$and $\pi(f)=\mathrm{id}_{H_{*}} \forall \pi \in t^{-1}(L) \subseteq \hat{G}$, hence $f a=a$, hence $a \in K_{G}^{+\infty} \subseteq K_{G}$.

Let us check $\pi(f)=\operatorname{id}_{H_{*}} \forall \pi \in t^{-1}(L)$. Let $\pi \in \hat{G}, \varphi$ with $\pi_{\varphi}=\pi$ :

$$
\begin{aligned}
\hat{f}\left(\left(\varphi \mid G_{F}\right)^{G}\right) & =\int_{G_{F}} f(x)\left(\varphi \mid G_{F}\right)^{G}(x) d x=\int_{G_{F}} f^{G}(x) \varphi(x) d x \\
& =\int_{G} f(x) \varphi(x) d x=\int_{G} f(x)\left(\pi(x) \xi_{\varphi}, \xi_{\varphi}\right) d x \\
& =\left(\pi(f) \xi_{\varphi}, \xi_{\varphi}\right) .
\end{aligned}
$$

Since $f$ is central and $\pi$ irreducible, $\pi(f)$ is a multiple of $\mathrm{id}_{H_{z}}$, so we have

$$
\pi(f)=\hat{f}\left(\left(\varphi \mid G_{F}\right)^{G}\right) \cdot \mathrm{id}_{H_{*}} \quad \forall \pi \in \hat{G}, \varphi \text { with } \pi_{\varphi}=\pi ;
$$

hence the assertion.

REMARK. In a SIN-group $G$ each quasicompact subset of $\hat{G}$ is contained in a closed quasicompact subset of $\hat{G}$ (because the mapping $p$ in the proof of Theorem 2 is continuous and proper).

LEMma. $G \in[\mathrm{SIN}], I$ a dense ideal in $L^{1}(G)$. Then for every quasicompact set $L \subseteq$ Prim $C^{*}(G)$ there is a $u \in I$ such that $u$ is a unit for $L^{1}(G)$ modulo $k(L) \cap$ $L^{1}(G) .\left[k(L):=\cap\left\{P \in \operatorname{Prim} C^{*}(G): P \in L\right\}\right]$. 
Proof. For $L$ quasicompact take $f$ as in the proof of Theorem 2; then $f$ is a unit for $L^{1}(G) /\left(k(L) \cap L^{1}(G)\right)$. Since $k(L) \cap L^{1}(G)$ is a modular ideal in $L^{1}(G)$, $I+\left(k(L) \cap L^{1}(G)\right)=L^{1}(G)[14,2.6 .8]$ and thus there is a $d \in k(L) \cap L^{1}(G)$ and $u \in I$ with $d+u=f$, hence $u-f \in k(L) \cap L^{1}(G)$ and thus $u$ is a unit for $L^{1}(G)$ modulo $\left(k(L) \cap L^{1}(G)\right)$.

Theorem 3. Let $G \in[S I N]$. Then there is a smallest dense ideal $J$ in $L^{1}(G) . J$ coincides with the intersection of $L^{1}(G)$ and the Pedersen ideal $K_{G}$ of $C^{*}(G)$ and also with the set of all $h \in L^{1}(G)$ for which the "Fourier transform" $\hat{h}$ vanishes outside a quasicompact subset of $\hat{G}$.

Proof. $J:=L^{1}(G) \cap K_{G}$ is dense in $L^{1}(G)$. Now let $I$ be an arbitrary dense ideal in $L^{1}(G)$. For every $a \in J$ there exists a quasicompact set $L \subseteq$ Prim $C^{*}(G)$ with $L \supseteq\left\{P \in\right.$ Prim $\left.C^{*}(G) ; a \notin P\right\}$, hence by the lemma above there is a $u \in I$ with $u a+P=a+P \forall P \in L$, and for all $P \in \operatorname{Prim} C^{*}(G) \backslash L$ too (since then $a \in P$ ). Thus we have $u a=a$, hence $a \in I$.

The last assertion follows from Theorem 2.

4. Added in proof. 1 . Let $G=G_{4,9}(0)$ be the group of all $3 \times 3$ matrices of the form

$$
\left[\begin{array}{ccc}
1 & x & z \\
0 & e^{r} & y \\
0 & 0 & 1
\end{array}\right], \quad r, x, y, z \in \mathbf{R} .
$$

Then the intersection of all dense two-sided ideal in $L^{1}(G)$ is trivial. The intersection of the Pedersen ideal of $C^{*}(G)$ with $L^{1}(G)$ is trivial, too. (Communicated by Viktor Losert.)

2. Let $G$ be a locally compact $T_{2}$ group. If $G_{0} \in$ [IN] $\left(G_{0}\right.$ the identity component) or if $G$ is a group of polynomial growth with symmetric group algebra $L^{1}(G)$, then there does exist a smallest dense two-sided ideal of $L^{1}(G)(V$. Losert, resp., J. Ludwig).

\section{REFERENCES}

1. J. Dixmier, Opérateurs de rang fini dans les représentations unitaires, Inst. Hautes Études Sci. Publ. Math. No. 6 (1960), 13-25.

2. E. Kaniuth, Topology in duals of SIN-groups, Math. Z. 134 (1973), 67-80.

3. E. Kaniuth and D. Steiner, On complete regularity of group algebras, Math. Ann. 204 (1973), 305-329.

4. E. Kotzmann, V. Losert and H. Rindler, Dense ideals of group algebras, Math. Ann. 246 (1979), $1-14$.

5. K. B. Laursen and A. M. Sinclair, Lifting matrix units in $C^{*}$-algebras. II, Math. Scand. 37 (1975), $167-172$.

6. J. Liukkonnen and R. Mosak, Harmonic analysis and centers of group algebras, Trans. Amer. Math. Soc. 195 (1974), 147-163.

7. J. Ludwig, A class of symmetric and a class of Wiener group algebras, J. Funct. Anal. 31 (1979), 187-194.

8. __ Polynomial growth and ideals in group algebras, Manuscripta Math. 30 (1980), 215-222.

9. R. Mosak, The $L^{1}$ - and $C^{*}$-algebras of $[\mathrm{FIA}]_{B}^{-}$-groups, and their representations, Trans. Amer. Math. Soc. 163 (1972), 277-310. 
10. T. W. Palmer, Classes of nonabelian, noncompact, locally compact groups, Rocky Mountain J. Math. 8 (1978), 683-741.

11. G. K. Pedersen, Measure theory for $C^{*}$-algebras, Math. Scand. 19 (1966), 131-145.

12. $\ldots, C^{*}$-integrals, an approach to noncommutative measure theory, Doctoral Thesis, Copenhagen Univ., Copenhagen, 1972.

13. F. Perdrizet, Topologie et traces sur les $C^{*}$-algèbres, Bull. Soc. Math. France 99 (1971), 193-239.

14. C. E. Rickart, General theory of Banach algebras, Krieger, Huntington, N. Y., 1974. (Reprint)

FACHbereich 17 (MAThematix/Informatix), Universttät-Gesamthochschule Paderborn, WARburger Strasse 100, D-4790 Paderborn, Federal Republic of Germany 